\title{
Effect and mechanism of action of SLP-2 on the apoptosis and autophagy of gastric cancer cells
}

\author{
SHENGSEN YANG, YUN HUANG, HONGYAN ZHANG, FANG WANG, LIANGUI SHAO and XUEHONG WANG
}

Department of Gastroenterology, Affiliated Hospital of Qinghai University, Xining, Qianghai 810001, P.R. China

Received February 8, 2021; Accepted May 28, 2021

DOI: 10.3892/ol.2021.12968

\begin{abstract}
This study was designed to investigate the effect of stomatin-like protein 2 (SLP-2) on the apoptosis and autophagy of gastric cancer cells and its underlying mechanism. The expression of SLP-2 was detected in human gastric cancer cell lines (AGS, MKN-45 and NCI-N87) and a human gastric epithelial cell line (GES-1) using reverse transcription-quantitative PCR and western blot analysis. SLP-2-specific small interfering RNA (siRNA) was transfected into NCI-N87 cells. Cell Counting Kit- 8 was used to detect cell proliferation. Apoptosis rates were measured using flow cytometry. Autophagosomes were observed by transmission electron microscopy. The expression levels of Annexin A2 (ANXA2), $\beta$-catenin, Bcl-2, Bax, Beclin-1 and LC3-II/I were also measured. The results demonstrated that SLP-2 siRNA transfection significantly reduced cell proliferation and increased cell apoptosis. The mitochondria were severely damaged, and a large number of autophagosomes were seen in SLP-2 siRNA-transfected NCI-N87 cells. Furthermore, the expression levels of ANXA2, $\beta$-catenin and Bcl-2 were downregulated, whereas those of Bax, Beclin-1 and LC3-II/I were upregulated following SLP-2 siRNA transfection. In conclusion, SLP-2 silencing can inhibit proliferation and induce apoptosis and autophagy of gastric cancer cells, and this effect may be related to the inhibition of ANXA2/ $\beta$-catenin signaling pathway.
\end{abstract}

\section{Introduction}

Gastric cancer is a common malignant tumor of the digestive system. According to the report released by the World Health Organization International Agency for Research on Cancer, there were 9.6 million cancer deaths and 18.1 million new cancer cases in 2018 worldwide, and gastric cancer ranks third

Correspondence to: Dr Xuehong Wang, Department of Gastroenterology, Affiliated Hospital of Qinghai University, 29 Tongren Road, Chengxi, Xining, Qinghai 810001, P.R. China E-mail: w13639718580@126.com

Key words: stomatin-like protein 2, gastric cancer, autophagy, apoptosis, ANXA2/ $\beta$-catenin signaling pathway with respect to mortality and fifth in terms of incidence (1). The incidence and mortality rates of gastric cancer are also high in China, which are about 2-fold higher than the global average $(1,2)$. Several genes, including tumor suppressors and oncogenes, are involved in the tumorigenesis and progression of gastric cancer. Overexpression, amplification and rearrangement of oncogenes can lead to the abnormal activation of cell signaling pathways, promote cell proliferation and differentiation, inhibit cell apoptosis and enhance cell invasion and metastasis $(3,4)$. Stomatin-like protein 2 (SLP-2) is a plasma membrane-associated protein originally identified in 2000 (5). It belongs to the highly conserved stomatin protein family and is expressed in several human tissue types, such as the stomach, lung, intestine, spleen and gallbladder $(5,6)$. Previous studies have shown that SLP-2 is upregulated and acts as an oncogene in a variety of human cancer types, including lung, endometrial, breast and colon cancer (7-9). However, there are few reports on the expression and the role of SLP-2 in gastric cancer. To the best of our knowledge, only one study has revealed that SLP-2 was upregulated in gastric cancer tissues compared with the adjacent-normal gastric epithelium, which suggested that high-level SLP-2 expression may contribute to the progression and poor prognosis of gastric cancer (7).

The aim of the present study was to examine the expression levels of SLP-2 in gastric cancer cell lines and to investigate the effect and mechanism of action of SLP-2 on the apoptosis and autophagy of gastric cancer cells. The findings may provide insight into the clinical treatment of gastric cancer.

\section{Materials and methods}

Cell lines and cell culture. The normal gastric mucosa cell line GES-1 (cat. no. BNCC353464), gastric adenocarcinoma cell line AGS (cat. no. BNCC309318) and MKN-45 (cat. no. BNCC337682) and gastric tubular adenocarcinoma cell line NCI-N87 (cat. no. BNCC312834) were purchased from BNBIO. NCI-N87, GES-1 and MKN-45 cells were cultured in RPMI-1640 medium (cat. no. KGM31800S; Nanjing KeyGen Biotech Co., Ltd.), whereas AGS cells were cultured in F12 medium (cat. no. KGM21700S; Nanjing KeyGen Biotech Co., Ltd.), containing 10\% FBS (Gibco; Thermo Fisher Scientific, Inc.) and $100 \mathrm{U} / \mathrm{ml}$ penicillin and streptomycin. The cells were maintained at $37^{\circ} \mathrm{C}$ in a humidified atmosphere with $5 \% \mathrm{CO}_{2}$. 
Cell transfection. Small interfering RNA (siRNA) against SLP-2 (siRNA-1, -2 and -3) and an siRNA negative control (NC) were purchased from ZHBY Biotech Co., Ltd and were transfected into NCI-N87 cells using Lipofectamine ${ }^{\mathrm{TM}} 3000$ transfection reagent (cat. no. L3000015; Invitrogen; Thermo Fisher Scientific, Inc.) according to the manufacturer's protocol. The sequences of all siRNAs used in the present study are as follows: SLP-2 siRNA-1 sense, 5'-GUGCAGAGUCUCAAG GAAATT-3' and antisense, 5'-UUUCCUUGAGACUCUGCA CTT-3';SLP-2siRNA-2sense,5'-AGAAAAGGCUGAACAGAU ATT-3' and antisense, 5'-UAUCUGUUCAGCCUUUUCUTT-3'; SLP-2 siRNA-3 sense,5'-GGCCAAGGCUAAAGCUGAATT-3' and antisense, 5'-UUCAGCUUUAGCCUUGGCCTT-3'; and siRNA NC sense, 5'-UUCUCCGAACGUGUCACGUTT-3' and antisense, 5'-ACGUGACACGUUCGGAGAATT-3' (reverse). Briefly, the cell culture medium was replaced with serum-free medium before transfection. A total of $2.5 \mu \mathrm{g}$ siRNA or $5 \mu \mathrm{l}$ Lipofectamine ${ }^{\circledR} 3000$ was separately diluted in $125 \mu \mathrm{l}$ of Opti-MEM (Gibco; Thermo Fisher Scientific, Inc.), then incubated at room temperature for $5 \mathrm{~min}$. The diluted plasmids were then added to the diluted Lipofectamine 3000 and incubated at room temperature for $15 \mathrm{~min}$. The complex was added to the cells and incubated at $37^{\circ} \mathrm{C}$ for $4 \mathrm{~h}$. Subsequently, the cell culture medium were replaced with fresh pre-warmed medium. After incubation for $48 \mathrm{~h}$, the cells were harvested for reverse transcription-quantitative (RT-qPCR) and western blot analysis.

$R T$ - $q P C R$. Total RNA was extracted from the cells using the Ultrapure RNA kit (cat. no. CW0581M; CWBio). The concentration and the purity [optical density (OD)260/OD280] of the RNA were determined using an NP80 UV-Vis spectrophotometer (NanoPhotometer; Implen $\mathrm{GmbH}$ ). The RNA was then reverse-transcribed to cDNA using the HiScript II Q RT SuperMix for qPCR with gDNA wiper (cat. no. R223-01; Vazyme Biotech Co., Ltd.). The reverse transcription reaction was conducted at $50^{\circ} \mathrm{C}$ for $15 \mathrm{~min}$, and then $85^{\circ} \mathrm{C}$ for $5 \mathrm{sec}$. The qPCR step was performed using 2X SYBR-Green PCR Master Mix (cat. no. A4004M; Xiamen Life Internet Technology Co., Ltd.) on a CFX connect ${ }^{\mathrm{TM}}$ Real-Time PCR detection system (Bio-Rad Laboratories, Inc.). The reaction was set up follows: i) RNase-free distilled $\mathrm{H}_{2} \mathrm{O}, 9.5 \mu \mathrm{l}$; ii) cDNA, $1 \mu \mathrm{l}$; iii) forward primer, $1 \mu \mathrm{l}$; iv) reverse primer, $1 \mu \mathrm{l}$; and v) $2 \mathrm{X}$ SYBR-Green PCR Master Mix, $12.5 \mu 1$. The primers were obtained from Anhui General Biosystems Co., Ltd., and their sequences are shown in Table I. The thermocycling conditions were included a pre-denaturation at $95^{\circ} \mathrm{C}$ for $10 \mathrm{~min}$ followed by 40 cycles of denaturation at $95^{\circ} \mathrm{C}$ for $10 \mathrm{sec}$, annealing at $57-62^{\circ} \mathrm{C}$ for $30 \mathrm{sec}$ and extension at $72^{\circ} \mathrm{C}$ for $30 \mathrm{sec}$. GAPDH was used as the internal control. The relative expression levels of SLP-2, ANXA2, $\beta$-catenin, Bcl-2 and Beclin-1 were calculated using the $2-\Delta \Delta$ Cq method (10).

Western blot analysis. RIPA cell lysis buffer (cat. no. C1053; Applygen Technologies, Inc.) was used to extract total protein from NCI-N87 cells. Protein concentration was measured using a BCA Assay kit (cat. no. CW0014S; CWBio). A total of $30 \mu \mathrm{g}$ protein was loaded per lane, separated by $10 \%$ SDS-PAGE, and then transferred onto nitrocellulose membranes at $300 \mathrm{~mA}$ for 80 min. After blocking with 5\% non-fat milk (cat. no. P1622; Applygen Technologies, Inc.) at $4^{\circ} \mathrm{C}$ overnight, the membranes were incubated at $4{ }^{\circ} \mathrm{C}$ overnight with primary antibodies (dilution, 1:500), including rabbit anti-SLP-2 (cat. no. 10348-1-AP; ProteinTech Group, Inc.), rabbit anti-Annexin A2 (ANXA2; cat. no. 11256-1-AP; ProteinTech Group, Inc.), rabbit anti-Bax (cat. no. 50599-2-lg; ProteinTech Group, Inc.), rabbit anti-Beclin-1 (cat. no. 11306-1-AP; ProteinTech Group, Inc.), rabbit anti- $\beta$-catenin (cat. no. ab32572; Abcam), mouse anti-Bcl-2 (cat. no. ab692; Abcam), rabbit anti-LC3-I/LC3-II (cat. no. bS-8878R; Biosynthesis Biotechnology Inc.) and mouse anti-GAPDH (cat. no. TA-08; ZSGB-BIO). The membranes were washed, then incubated with horseradish peroxidase-conjugated secondary antibodies at 1:1,000 dilution, including goat anti-mouse $\operatorname{IgG}(\mathrm{H}+\mathrm{L})$ (cat. no. ZB-2305; ZSGB-BIO) and goat anti-rabbit $\operatorname{IgG}(\mathrm{H}+\mathrm{L})$ (cat. no. ZB-2301; ZSGB-BIO) at room temperature for $2 \mathrm{~h}$. The band signals were developed using ECL solution (Thermo Fisher Scientific, Inc.). The Chemi Doc ${ }^{\mathrm{TM}}$ XRS+ system (Bio-Rad Laboratories, Inc.) was used for visualization. Protein expression was quantified by using ImageJ software (version 18.0; National Institutes of Health).

Cell Counting Kit-8 (CCK-8). Cells were seeded into 96-well plates $\left(4 \times 10^{4}\right.$ cells per well), and cell proliferation was detected using a CCK-8 kit according to the manufacturer's instructions. The cells in each well were incubated with $10 \mu \mathrm{l} \mathrm{CCK}-8$ reagent at $37^{\circ} \mathrm{C}$ for $2 \mathrm{~h}$. The OD was detected in each well at $450 \mathrm{~nm}$ using a WD-2102B microplate reader (Beijing Liuyi Biotechnology Co., Ltd.).

Flow cytometry analysis of apoptosis. Early and late apoptosis was detected using an Annexin V-FITC/PI Apoptosis Kit (cat. no. AP101-100; Multi Sciences Lianke Biotech, Co., Ltd.). A total of $1-3 \times 10^{6}$ cells were collected and centrifuged with $1 \mathrm{ml}$ PBS (twice at $503 \times \mathrm{g}, 4^{\circ} \mathrm{C}$ for $3 \mathrm{~min}$ ) The cells were resuspended in $1.5 \mathrm{ml}$ Binding Buffer and added to three tubes (one for the the blank control, the other two as single staining with $5 \mu \mathrm{l}$ Annexin V-FITC or $10 \mu \mathrm{l}$ PI-PE solution), and incubated at room temperature for $10 \mathrm{~min}$. The blank control tube was used to adjust the voltage of FSC, SSC and the fluorescence channel. Under these voltage conditions, the single-stained tube was used to adjust the compensation of the fluorescence channel. After adjusting the parameters, the samples were resuspended in Binding Buffer, and $5 \mu \mathrm{l}$ Annexin V-FITC and $10 \mu 1 \mathrm{PI}-\mathrm{PE}$ solution were added. After incubation at room temperature for $10 \mathrm{~min}$ in the dark, $200 \mu \mathrm{l}$ ice-cold $1 \mathrm{X}$ Binding Buffer was added and mixed well. Flow cytometric data were acquired using a NovoCyte ${ }^{\mathrm{TM}}$ 2060R flow cytometer (ACEA Biosciences) with NovoExpress software (version 1.2.5; Agilent Technologies, Inc.).

Transmission electron microscopy of autophagosomes. The cells were fixed in $2.5 \%$ glutaraldehyde at $4^{\circ} \mathrm{C}$ overnight, and then washed three times with cold PBS. After fixing in $1 \%$ osmic acid at $4^{\circ} \mathrm{C}$ for $1 \mathrm{~h}$, and dehydration with gradient alcohol and acetone, the cells were embedded in epoxy resin overnight at room temperature, and placed in an oven at $60^{\circ} \mathrm{C}$ for $2 \mathrm{~h} .60-\mathrm{nm}$ sections were cut from the blocks, and then double-stained with $2 \%$ uranyl acetate and lead citrate at room temperature for 5-10 min. The autophagosomes were observed under a transmission electron microscope (HT7700; Hitachi, Ltd.). 
Table I. Primer sequences and annealing temperatures.

\begin{tabular}{|c|c|c|c|c|}
\hline Primer name & Primer sequences, 5'-3' & $\begin{array}{l}\text { Primer } \\
\text { length, bp }\end{array}$ & $\begin{array}{l}\text { Product } \\
\text { length, bp }\end{array}$ & $\begin{array}{l}\text { Annealing } \\
\text { temperature, }{ }^{\circ} \mathrm{C}\end{array}$ \\
\hline SLP-2 & F: GCTGCTGACTGCTGGGGTAT & 20 & 236 & 60.5 \\
\hline SLP-2 & R: CCTGCTGCCTGATTTATCTGTT & 22 & & \\
\hline ANXA2 & F: ACCTGGAGACGGTGATTTTG & 20 & 263 & 58.1 \\
\hline ANXA2 & R: CTCTGCTCTTCTACCCTTTGC & 21 & & \\
\hline$\beta$-catenin & F: TCTGAGGACAAGCCACAAGAT & 21 & 109 & 60.0 \\
\hline$\beta$-catenin & R: CAAGTCCAAGATCAGCAGTCTCATT & 25 & & \\
\hline Bcl-2 & F: TGAGTTCGGTGGGGTCAT & 18 & 189 & 57.3 \\
\hline Bcl-2 & R: CAGGAGAAATCAAACAGAGGC & 21 & & \\
\hline Bax & F: GGATGCGTCCACCAAGAA & 18 & 193 & 57.0 \\
\hline Bax & R: AAAGTAGAAAAGGGCGACAAC & 21 & & \\
\hline Beclin-1 & F: AATAACTTCAGGCTGGGTCG & 20 & 141 & 58.2 \\
\hline Beclin-1 & R: AGGAACAAGTCGGTATCTCTGAA & 23 & & \\
\hline GAPDH & F: TGACTTCAACAGCGACACCCA & 21 & 121 & 61.5 \\
\hline GAPDH & R: CACCCTGTTGCTGTAGCCAAA & 21 & & \\
\hline
\end{tabular}

SLP-2, stomatin-like protein 2; ANXA2, Annexin A2; F, forward; R, reverse; bp, base pair.

Statistical analysis. GraphPad Prism7 software (GraphPad Software, Inc.) was used for statistical analysis. The results are presented as the mean \pm standard deviation. Differences between groups were evaluated using one-way ANOVA followed by the Least Significant Difference test and Tukey's post hoc tests. $\mathrm{P}<0.05$ was considered to indicate a statistically significant difference.

\section{Results}

Expression of SLP-2 in gastric cancer cell lines. In order to select the gastric cancer cell line with the highest expression levels of SLP-2, RT-qPCR and western blot analysis were used to measure SLP-2 expression in different gastric cancer cell lines (Fig. 1). Compared with the normal cell line GES-1, the mRNA and protein expression levels of SLP-2 significantly increased in NCI-N87 cell line, but did not change significantly in AGS cell line. SLP-2 mRNA expression level was decreased, but its protein expression level was increased in the MKN-45 cell line. Therefore, NCI-N87 cell line was selected for subsequent experiments.

Efficiency of SLP-2 siRNA-mediated silencing . In order to inhibit SLP-2 expression, three SLP-2 specific siRNA candidates were designed and transfected into NCI-N87 cells. The silencing efficiency of these siRNA molecules was detected using RT-qPCR and western blot analysis (Fig. 2). Compared with the control and siRNA NC groups, siRNA-1, siRNA-2 and siRNA-3 significantly decreased the mRNA and protein expression levels of SLP-2 in NCI-N87 cells. siRNA-2 was chosen for subsequent, as it displayed the highest efficiency of SLP-2 silencing.

Effect of siRNA SLP-2 on the proliferation and apoptosis of NCI-N87 cells. In order to examine the effect of siRNA
SLP-2 on the proliferation of NCI-N87 cells, CCK-8 was used to detect cell proliferation (Fig. 3A). Compared with the control and siRNA NC groups, siRNA SLP-2 significantly inhibited the proliferation of NCI-N87 cells. In addition, the rate of early and late apoptosis was evaluated using flow cytometry (Fig. 3B). Compared with the control and siRNA $\mathrm{NC}$ groups, the apoptotic rate significantly increased in the siRNA SLP-2 group.

Effect of siRNA SLP-2 on the expression of ANXA2, $\beta$-catenin, Bcl-2 and Bax in NCI-N87 cells. RT-qPCR and western blot analysis were used to detect the expression levels of ANXA2, $\beta$-catenin, Bcl-2 and Bax in NCI-N87 cells (Fig. 4). Compared with the control and siRNA NC groups, the mRNA and protein expression levels of ANXA2, $\beta$-catenin and Bcl- 2 were significantly reduced in the siRNA SLP-2 group, whereas the expression of Bax was significantly increased.

Effect of siRNA SLP-2 on autophagy of NCI-N87 cells. To investigate the effect of siRNA SLP-2 on the autophagy of NCI-N87 cells, autophagosomes were observed by transmission electron microscopy, and the expression of Beclin-1 and LC3-II/I was detected by western blot analysis. As shown in Fig. 5A, compared with the control and siRNA NC groups, the mitochondria were severely damaged, and a large number of autophagosomes were observed in the siRNA SLP-2 group. Furthermore, the protein levels of Beclin-1 and LC3-II/I in the siRNA SLP-2 group were significantly increased (Fig. 5B). These results indicated that siRNA SLP-2 can induce autophagy in NCI-N87 cells.

\section{Discussion}

In the present study, the expression of SLP-2 was examined in human gastric cancer cell lines (AGS, MKN-45 and NCI-N87) 

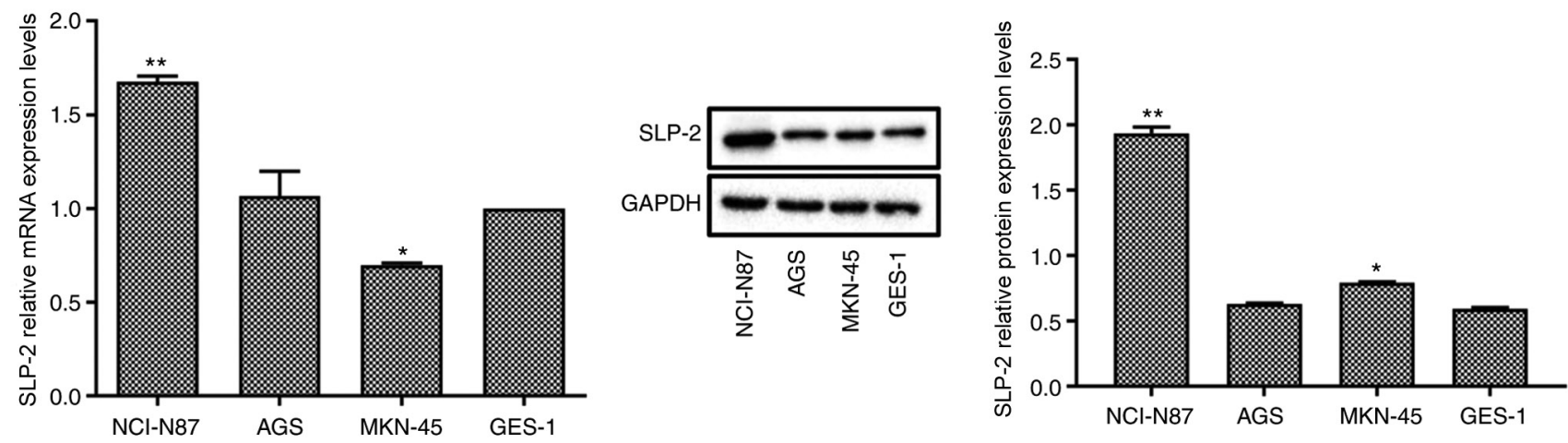

Figure 1. Expression of SLP-2 in the human gastric cancer cell lines AGS, MKN-45 and NCI-N87 and the human gastric epithelial cell line GES-1. $\mathrm{n}=6$. Differences between groups were evaluated using one-way ANOVA followed by Tukey's post hoc test. ${ }^{*} \mathrm{P}<0.05$ and ${ }^{* *} \mathrm{P}<0.01$ vs. GES-1. SLP-2, stomatin-like protein 2.
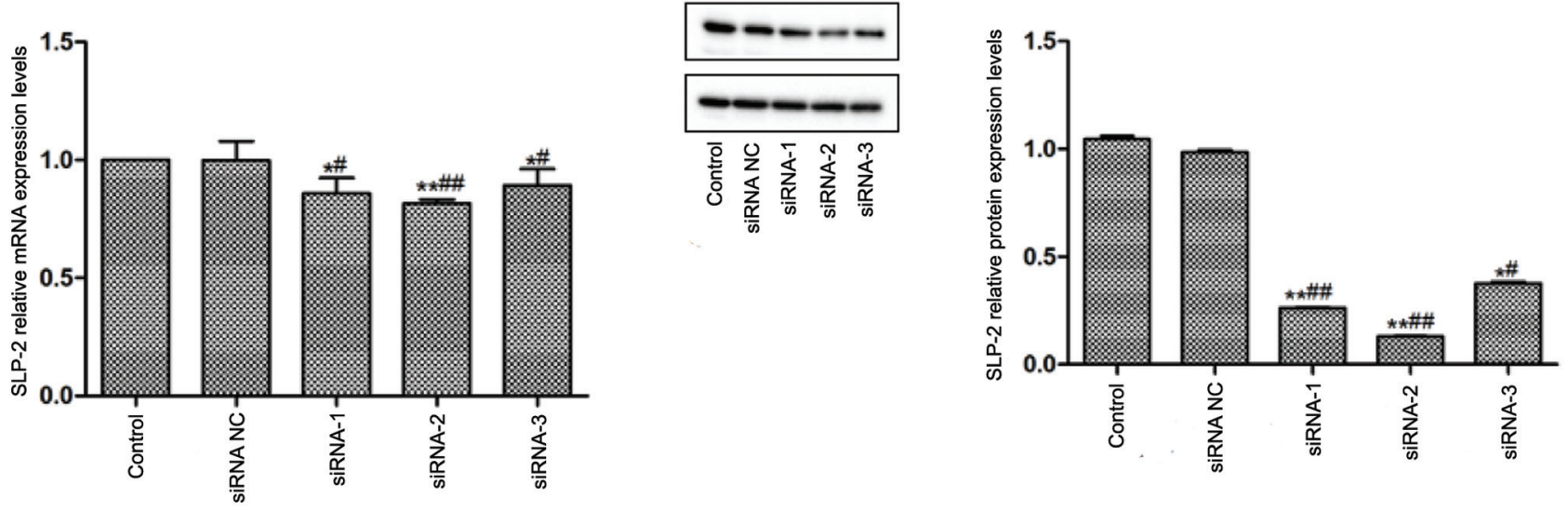

Figure 2. Expression of SLP-2 in NCI-N87 cells following transfection with SLP-2 siRNA. $n=6$. Differences between groups were evaluated using one-way ANOVA followed by Tukey's post hoc tests. ${ }^{*} \mathrm{P}<0.05$ and ${ }^{* * *} \mathrm{P}<0.01$ vs. control; ${ }^{*} \mathrm{P}<0.05$ and ${ }^{\# \#} \mathrm{P}<0.01$ vs. siRNA NC. SLP-2, stomatin-like protein 2 ; siRNA, small interfering RNA; NC, negative control.

and a human gastric epithelial cell line (GES-1). The AGS cell line was originally derived from the resected tumor of a patient with gastric adenocarcinoma (11). The NCI-N87 cell line was derived from the metastatic site of a patient with gastric carcinoma (11). In the present study, SLP-2 expression level was higher in a cell line originating from distant metastases (NCI-N87) than in the cell line derived from a primary tumor (AGS), indicating that SLP-2 exhibits differential expression in different stages of gastric cancer, and that SLP-2 may be associated with the pathogenesis of gastric cancer. Since the NCI-N87 cell line expressed the highest levels of SLP-2 among all the tested cell lines, it was used for subsequent loss-of-function experiments.

SLP-2 is a membrane-associated protein first isolated from human erythrocytes by Wang and Morrow in 2000 (5). Later, Wang et al (12) demonstrated that SLP-2 was also a mitochondrial protein that played important roles in maintaining calcium homeostasis (12). A number of studies have demonstrated that SLP-2 is highly expressed in tumor tissue and is closely related to the occurrence and development of tumors (7-9). For instance, Zhang et al (13) reported that SLP-2 was overexpressed in ovarian cancer tissue and that its expression correlated with clinical stage, pathological differentiation and lymph node metastasis of patients with ovarian cancer, suggesting that SLP-2 may play a role in promoting the invasion and metastasis of ovarian cancer. Moreover, Zhou et al suggested that SLP-2 was highly expressed in colorectal cancer tissues and cell lines and that positive expression of SLP-2 could be used as an independent risk factor for poor prognosis of colorectal cancer (14). Silencing of the SLP-2 gene significantly inhibits the proliferation, migration and invasion of colorectal cancer cells (14). Liu et al detected the expression of SLP-2 in primary gastric cancer tissue and matched normal gastric tissue samples using RT-qPCR and western blot analysis, which indicated that SLP-2 was highly expressed in gastric cancer tissues (7). Furthermore, they observed that high expression of SLP-2 was closely related to the depth of invasion, lymph node metastasis, distant metastasis and American Joint Committee on Cancer stage. Cox regression analysis also indicated that the expression of SLP-2 was an independent prognostic factor for gastric cancer (7). These findings suggest that SLP-2 may be an oncogene involved in the development of gastric cancer.

Apoptosis is an active, sequential and programmed cell death process (15). Autophagy is a survival process in which eukaryotic cells degrade damaged or senescent organelles and proteins for cellular renewal (15). Crosstalk can occur between autophagy and apoptosis (16-18). The activation of autophagy usually protects cells from apoptosis $(15,19)$. In some circumstances, however, autophagy may promote cellular demise 

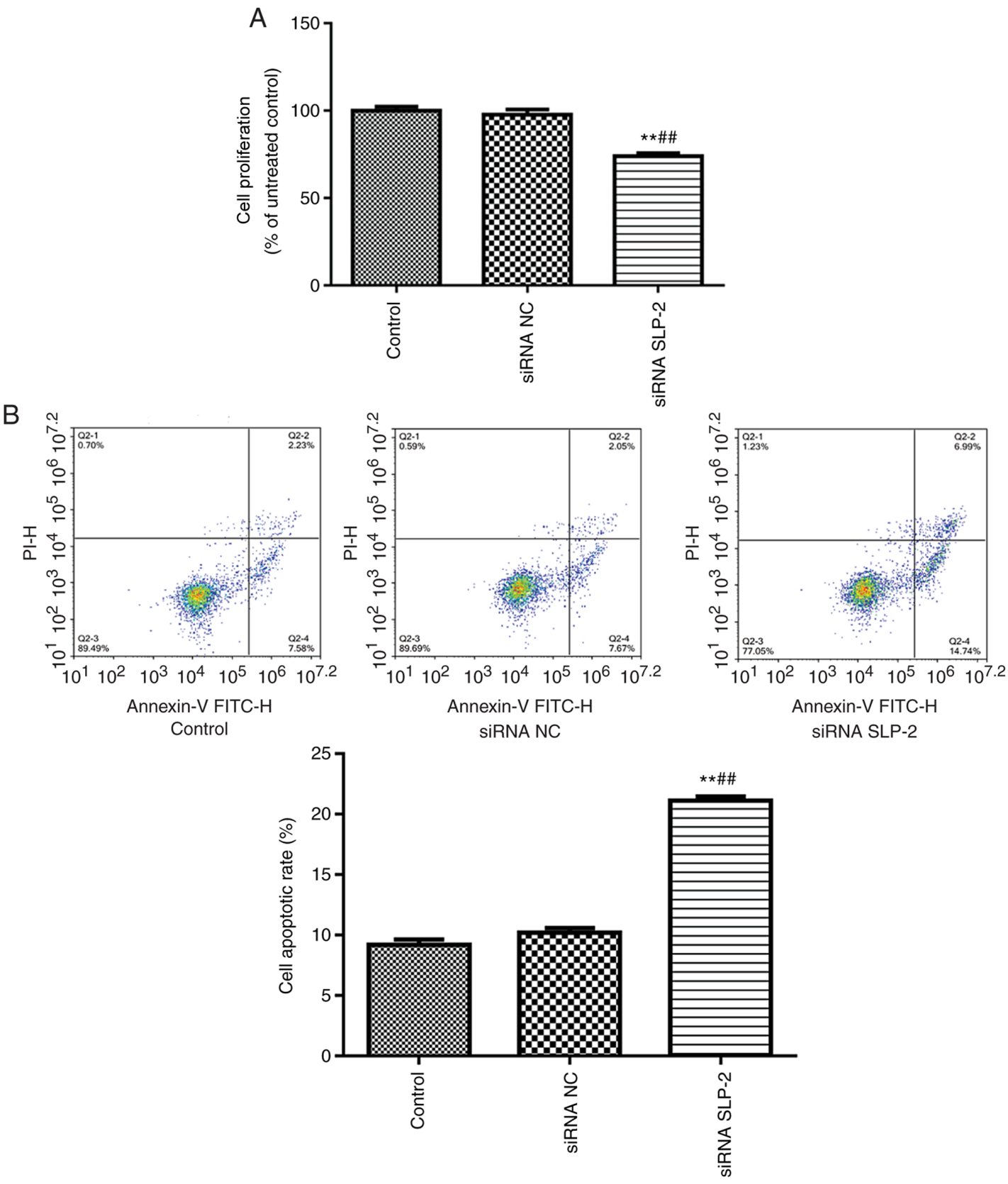

Figure 3. Effect of siRNA SLP-2 on the proliferation and apoptosis of NCI-N87 cells. Effect of siRNA SLP-2 on NCI-N87 cell (A) proliferation and (B) apoptosis. $n=6$. Differences between groups were evaluated using one-way ANOVA followed by the Least Significant Difference test. ${ }^{* *} \mathrm{P}<0.01$ compared with control; "\# $\mathrm{P}<0.01$ vs. siRNA NC. SLP-2, stomatin-like protein 2; siRNA, small interfering RNA; NC, negative control; PI, propidium iodide; FITC, fluorescein isothiocyanate.
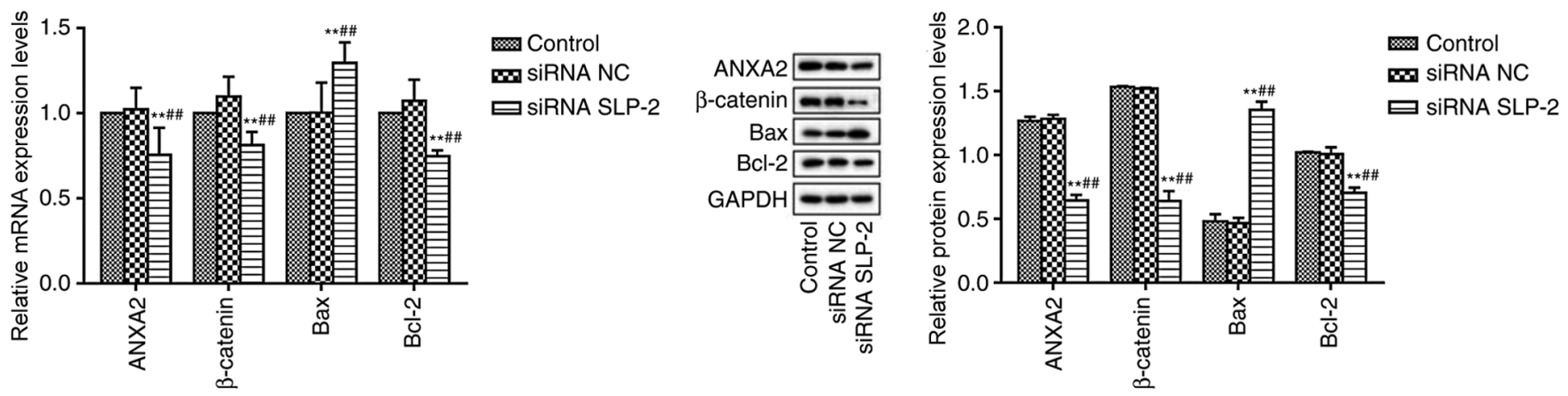

Figure 4. Effect of siRNA SLP-2 on the expression of ANXA2, $\beta$-catenin, Bcl-2 and Bax in NCI-N87 cells. $\mathrm{n}=6$. Differences between groups were evaluated using one-way ANOVA followed by the Least Significant Difference test. ${ }^{* *} \mathrm{P}<0.01$ vs. control, ${ }^{\# \#} \mathrm{P}<0.01$ vs. siRNA NC. SLP-2, stomatin-like protein 2; siRNA, small interfering RNA; NC, negative control; ANXA2, Annexin A2. 


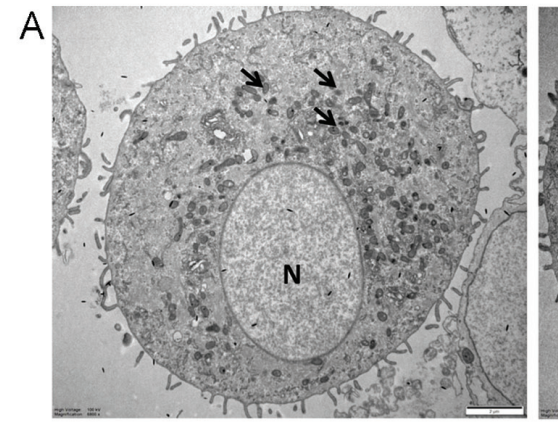

Control

B

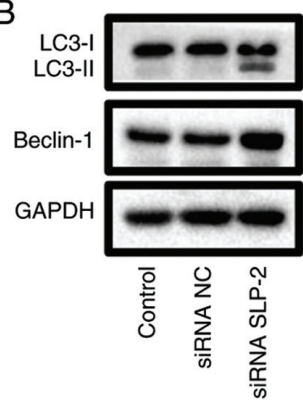

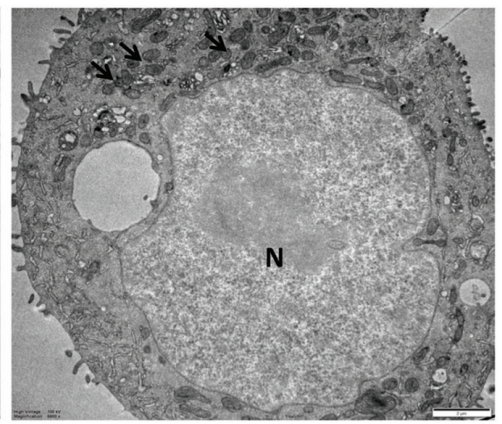

SIRNA NC

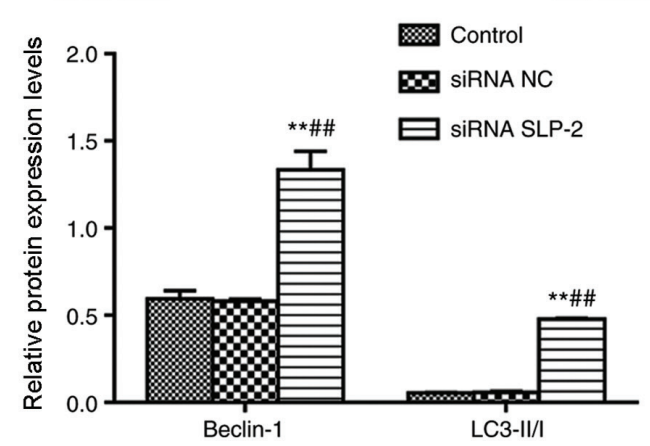

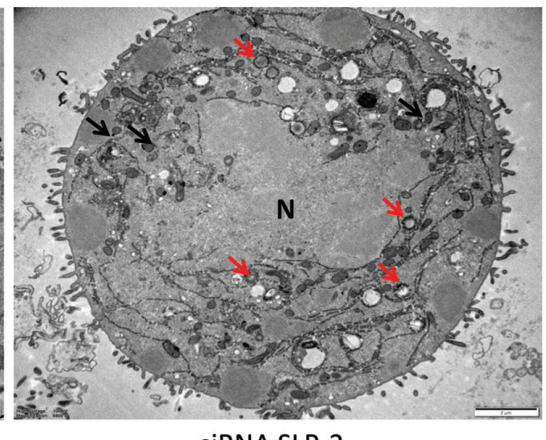

SIRNA SLP-2

Figure 5. Effect of siRNA SLP-2 on autophagy of NCI-N87 cells. (A) Autophagosomes were observed by transmission electron microscopy. (B) Expression of Beclin-1 and LC3-II/I was detected by western blotting. Scale bar, $2 \mu \mathrm{m}$. N, nucleus; black arrowhead, mitochondrion; red arrowhead, autophagosome. $\mathrm{n}=6$. Differences between groups were evaluated using one-way ANOVA followed by the Least Significant Difference test. ${ }^{* *} \mathrm{P}<0.01$ vs. control; ${ }^{\# \#} \mathrm{P}<0.01$ vs. siRNA NC. SLP-2, stomatin-like protein 2; siRNA, small interfering RNA; NC, negative control.

by inducing apoptosis $(15,19)$. Thus, the complex interplay between autophagy and apoptosis determines the fate of cells. The BCL-2 protein family is a central regulator of autophagy and apoptosis (20). The anti-apoptotic protein Bcl-2 can form a heterodimer with the pro-apoptotic protein Bax, resulting in the enhancement of the anti-apoptotic effect of Bcl-2 (21). Bax is also able to form homodimers to promote apoptosis (21). The autophagy-related protein Beclin-1 can competitively bind with Bcl-2/Bcl-xl to promote autophagy (21). The inhibition of apoptosis and the induction of autophagy have been documented in several types of tumor, including gastric cancer (15). In recent years, the development of autophagy inhibitors, as well as inducers of tumor cell apoptosis to a certain extent, has attracted increasing attention (16,22-24).

In the present study, CCK- 8 and flow cytometry were used to examine the effect of SLP-2 on the proliferation and apoptosis of NCI-N87 cells. The results demonstrated that downregulation of SLP-2 could significantly inhibit proliferation and induce apoptosis of NCI-N87 cells. In addition, RT-qPCR and western blot results indicated that SLP-2 silencing could significantly downregulate Bcl-2 and upregulate Bax in these cells. This finding is consistent with a previous study suggesting that downregulation of SLP-2 can inhibit the proliferation and induce the apoptosis of glioma cells by regulating the expression of Bcl-2 and Bax (25). To the best of our knowledge, the role of SLP-2 on autophagy has not been reported to date. Thus, this study is the first to reveal the effect of SLP-2 on autophagy of gastric cancer cells. Moreover, the present results showed a large number of autophagosomes in siRNA SLP-2-transfected cells, and siRNA SLP-2 upregulated Beclin-1 and LC3-I/II, suggesting that the downregulation of SLP-2 may induce apoptosis of NCI-N87 cells by promoting autophagy.
Apoptosis and autophagy are mediated by multiple signaling pathways, among which mTOR signaling pathway is the most important $(26,27)$. It has been reported that ANXA2 can regulate autophagy in Pseudomonas aeruginosa infection through the AKT1-mTOR-ULK1/2 signaling pathway (28). ANXA2 is a multifunctional calcium-dependent phospholipid-binding protein that is widely distributed in the membrane, cytoplasm and nucleus of eukaryotic cells (29). ANXA2 plays important roles in cytoskeleton remodeling, cell phenotype change and cell movement by regulating the expression of connexin molecules (30-32). The abnormal expression of ANXA2 is closely associated type 2 diabetes, cancer and autoimmune diseases (33-35). Yang et al (36) analyzed protein-protein interactions in SLP-2-overexpressing A549 cells using immunoprecipitation and proteomics and determined that ANXA2 interacted with SLP-2 and $\beta$-catenin directly. Additional experiments demonstrated that the knockout of the SLP-2 gene inhibited SLP-2/ANXA2/ $\beta$-catenin cascade formation, reduced the translocation of cytoplasmic $\beta$-catenin into the nucleus and suppressed the expression of the downstream target gene, survivin, thereby inhibiting the proliferation of non-small cell lung cancer cells (36). Zhou et al demonstrated that the cell viability, migration and invasion of SW620 significantly decreased following SLP-2 gene silencing (14). In addition, silencing of the SLP-2 gene in SW620 cells inhibited the expression of metastasis-associated genes and the activation of Wnt/ $\beta$-catenin signaling pathway (14). In the present study, the expression levels of ANXA2 and $\beta$-catenin were determined in NCI-N87 cells. The results demonstrated that SLP-2 silencing could significantly downregulate the expression of ANXA2 and $\beta$-catenin. This finding is consistent with a 
previous report in NSCLC cells (36) and suggests that SLP-2 may affect the apoptosis and autophagy of gastric cancer cells by regulating ANXA2/ $\beta$-catenin signaling.

A limitation of this study is that the regulatory effect of SLP-2 on the ANXA2/ $\beta$-catenin signaling pathway was only determined in NCI-N87 cells. In addition, ANXA2/ $\beta$-catenin signaling inhibitors could be used in future studies to confirm the hypothesis that this signaling pathway mediates the effect of SLP-2 on gastric cancer cell apoptosis and autophagy.

In conclusion, SLP-2 silencing significantly induced apoptosis and autophagy, as well as inhibited the proliferation of NCI-N87 cells, and this effect may be mediated by inhibition of ANXA2/ $\beta$-catenin signaling. These findings revealed the effect and the mechanism of action of SLP-2 in gastric cancer cells and provided new potential avenues for the clinical treatment of gastric cancer.

\section{Acknowledgements}

Not applicable.

\section{Funding}

This study was supported by The National Key Project of Precision Medicine Research (grant no. 2017YFC0908300) and The Qinghai Provincial Scientific Research Project (grant no. 2019-SF-L3).

\section{Availability of data and materials}

The data sets generated and analyzed during the present study are available from the corresponding author on reasonable request.

\section{Authors' contributions}

XW and SY designed the study and prepared the manuscript. SY, YH, HZ, FW and LS conducted the experiments and analyzed the data. All authors were substantially involved in the research, acquisition of data, analysis and manuscript preparation. All authors read and approved the final manuscript. XW and SY confirmed the authenticity of all the raw data.

\section{Ethics approval and consent to participate}

Not applicable.

\section{Patient consent for publication}

Not applicable.

\section{Competing interests}

The authors declare that they have no competing interests.

\section{References}

1. Bray F, Ferlay J, Soerjomataram I, Siegel RL, Torre LA and Jemal A: Global cancer statistics 2018: GLOBOCAN estimates of incidence and mortality worldwide for 36 cancers in 185 countries. CA Cancer J Clin 68: 394-424, 2018.
2. Li Z, Ying X, Shan F and Ji J: The association of garlic with Helicobacter pylori infection and gastric cancer risk: A systematic review and meta-analysis. Helicobacter 23: e12532, 2018.

3. Mao QQ, Xu XY, Shang A, Gan RY, Wu DT, Atanasov AG and $\mathrm{Li} \mathrm{HB}$ : Phytochemicals for the prevention and treatment of gastric cancer: Effects and Mechanisms. Int J Mol Sci 21: 21, 2020.

4. Xiao S and Zhou L: Gastric cancer: Metabolic and metabolomics perspectives (Review). Int J Oncol 51: 5-17, 2017.

5. Wang Y and Morrow JS: Identification and characterization of human SLP-2, a novel homologue of stomatin (band 7.2b) present in erythrocytes and other tissues. J Biol Chem 275: 8062-8071, 2000.

6. Deng H, Deng Y, Liu F, Chen J, Li Z, Zhao K, Guan X and Liang W: Stomatin-like protein 2 is overexpressed in cervical cancer and involved in tumor cell apoptosis. Oncol Lett 14: 6355-6364, 2017.

7. Liu D, Zhang L, Shen Z, Tan F, Hu Y, Yu J and Li G: Increased levels of SLP-2 correlate with poor prognosis in gastric cancer. Gastric Cancer 16: 498-504, 2013.

8. Yang X, Hu Y, Shi H, Zhang C, Wang Z, Liu X, Chen H, Zhang L and Cui D: The diagnostic value of TROP-2, SLP-2 and CD56 expression in papillary thyroid carcinoma. Eur Arch Otorhinolaryngol 275: 2127-2134, 2018

9. Liu Q, Li A, Wang L, He W, Zhao L, Wu C, Lu S, Ye X, Zhao H, Shen X, et al: Stomatin-like protein 2 promotes tumor cell survival by activating the JAK2-STAT3-PIM1 pathway, suggesting a novel therapy in CRC. Mol Ther Oncolytics 17: 169-179, 2020.

10. Livak KJ and Schmittgen TD: Analysis of relative gene expression data using real-time quantitative PCR and the 2(-Delta Delta C(T)) method. Methods 25: 402-408, 2001.

11. Burgermeister E, Xing X, Röcken C, Juhasz M, Chen J, Hiber M, Mair K, Shatz M, Liscovitch M, Schmid RM, et al: Differential expression and function of caveolin-1 in human gastric cancer progression. Cancer Res 67: 8519-8526, 2007.

12. Wang Y, Cao W, Yu Z and Liu Z: Downregulation of a mitochondria associated protein SLP-2 inhibits tumor cell motility, proliferation and enhances cell sensitivity to chemotherapeutic reagents. Cancer Biol Ther 8: 1651-1658, 2009.

13. Zhang J, Song X, Li C and Tian Y: Expression and clinical significance of SLP-2 in ovarian tumors. Oncol Lett 17: 4626-4632, 2019.

14. Zhou C, Li Y, Wang G, Niu W, Zhang J, Wang G, Zhao Q and Fan L: Enhanced SLP-2 promotes invasion and metastasis by regulating $\mathrm{Wnt} / \beta$-catenin signal pathway in colorectal cancer and predicts poor prognosis. Pathol Res Pract 215: 57-67, 2019.

15. Liu JZ, Hu YL, Feng Y, Guo YB, Liu YF, Yang JL, Mao QS and Xue WJ: Rafoxanide promotes apoptosis and autophagy of gastric cancer cells by suppressing PI3K/Akt/mTOR pathway. Exp Cell Res 385: 111691, 2019.

16. Wang G, Zhang T, Sun W, Wang H, Yin F, Wang Z, Zuo D, Sun M, Zhou Z, Lin B, et al: Arsenic sulfide induces apoptosis and autophagy through the activation of ROS/JNK and suppression of Akt/mTOR signaling pathways in osteosarcoma. Free Radic Biol Med 106: 24-37, 2017.

17. Nowak KL and Edelstein CL: Apoptosis and autophagy in polycystic kidney disease (PKD). Cell Signal 68: 109518, 2020.

18. Kasprowska-Liśkiewicz D: The cell on the edge of life and death: Crosstalk between autophagy and apoptosis. Postepy Hig Med Dosw 71: 825-841, 2017.

19. D'Arcy MS: Cell death: A review of the major forms of apoptosis, necrosis and autophagy. Cell Biol Int 43: 582-592, 2019.

20. Edlich F: BCL-2 proteins and apoptosis: Recent insights and unknowns. Biochem Biophys Res Commun 500: 26-34, 2018

21. Tang Q, Bu W, Wang D, Xin Y, Xu X and Sun H: Advance research on interaction between autophagy and apoptosis and its influence in development of tumors. J Jilin Univ 41: 1303-1306, 2015.

22. Cao Y, Luo Y, Zou J, Ouyang J, Cai Z, Zeng X, Ling H and Zeng T: Autophagy and its role in gastric cancer. Clin Chim Acta 489: 10-20, 2019.

23. Qian HR and Yang Y: Functional role of autophagy in gastric cancer. Oncotarget 7: 17641-17651, 2016.

24. Song J, Zhou Y, Gong Y, Liu H and Tang L: Rottlerin promotes autophagy and apoptosis in gastric cancer cell lines. Mol Med Rep 18: 2905-2913, 2018.

25. Chen X, Wang P, Xie M, Zhao H and Wu H: Study on mechanism of down-regulation of SLP-2 gene expression on proliferation and apoptosis of glioma cells. Chin J Immunol 34: 55-59, 2018. (In Chinese). 
26. Yu Z, Chen Y, Liang C and Eriocalyxin B: Eriocalyxin B Induces apoptosis and autophagy involving Akt/mammalian target of rapamycin (mTOR) pathway in prostate cancer cells. Med Sci Monit 25: 8534-8543, 2019.

27. Li Y, Wang C, Liu Y, You J and Su G: Autophagy, lysosome dysfunction and mTOR inhibition in MNU-induced photoreceptor cell damage. Tissue Cell 61: 98-108, 2019.

28. Li R, Tan S, Yu M, Jundt MC, Zhang S and Wu M: Annexin A2 regulates autophagy in Pseudomonas aeruginosa infection through the Akt1-mTOR-ULK1/2 signaling pathway. J Immunol 195: 3901-3911, 2015.

29. Karimi-Busheri F, Marcoux Y, Tredget EE, Li L, Zheng J, Ghoreishi M, Weinfeld $M$ and Ghahary A: Expression of a releasable form of annexin II by human keratinocytes. J Cell Biochem 86: 737-747, 2002.

30. Shi H, Xiao L, Duan W, He H, Ma L, Da M, Duan Y, Wang Q, Wu H, Song X, et al: ANXA2 enhances the progression of hepatocellular carcinoma via remodeling the cell motility associated structures. Micron 85: 26-33, 2016.

31. He H, Xiao L, Cheng S, Yang Q, Li J, Hou Y, Song F, Su X, Jin H Liu Z, et al: Annexin A2 enhances the progression of colorectal cancer and hepatocarcinoma via cytoskeleton structural rearrangements. Microsc Microanal 25: 950-960, 2019.
32. Chen CY, Lin YS, Chen CH and Chen YJ: Annexin A2-mediated cancer progression and therapeutic resistance in nasopharyngeal carcinoma. J Biomed Sci 25: 30, 2018

33. Sharma MC: Annexin A2 (ANX A2): An emerging biomarker and potential therapeutic target for aggressive cancers. Int J Cancer 144: 2074-2081, 2019.

34. Wang T, Wang Z, Niu R and Wang L: Crucial role of Anxa2 in cancer progression: Highlights on its novel regulatory mechanism. Cancer Biol Med 16: 671-687, 2019.

35. Cardoso CM, de Jesus SF, de Souza MG, Queiroz LD, Santos EM, Dos Santos EP, Oliveira LP, Santos CK, Sousa Santos SH, et al: High levels of ANXA2 are characteristic of malignant salivary gland tumors. J Oral Pathol Med 48: 929-934, 2019.

36. Yang CT, Li JM, Li LF, Ko YS and Chen JT: Stomatin-like protein 2 regulates survivin expression in non-small cell lung cancer cells through $\beta$-catenin signaling pathway. Cell Death Dis 9: 425,2018 International (CC BY-NC-ND 4.0) License. 